\section{A PL/I program to generate randomized stimulus and response sequences for experiments}

\section{CHARLES F. HOFACKER \\ University of California, Los Angeles, California 90024}

In many experiments on attitudes, memory, and personality, it is desirable to randomize the stimulus set presented to subjects. One solution to the randomization problem is to have each subject seated at a computer terminal. In experiments with large numbers of subjects, however, such a strategy becomes impractical. When paper is used for presentation or answer sheets, numerous versions of the sheets may be required, each with its own randomized version of the presentation and response stimuli. For example, in a memory experiment, it may be necessary to generate several randomized sequences of the stimuli to be memorized so that each group of subjects receives the stimuli in a different order. Sometimes, that which is ideal from the standpoint of randomization must be compromised due to the time and effort involved in randomizing materials. Answer sheets have the further disadvantage that scoring can be slow and error prone.

The current program, EXP, offers an alternative to the use of answer sheets or computer terminals. The program can be used to generate Hollerith (IBM) cards in decks. Each subject has his own deck with an individualized stimulus and response sequence. Information that identifies the experimental condition can be coded on each card but hidden from the subject. Keypunch machines can be programmed with a drum card to interpret only the part of the card that the experimenter would like the subject to see.

During the experiment, the subject marks his response on the card. When the experiment is over, the decks can be sorted into two or more piles. In a memory experiment, the piles might consist of wrong and right. In an experiment in which subjects are asked to give graded responses, there would be one pile for each possible response. Examples of such experiments include recognition or identification studies in which confidence judgments are taken, absolute identification tasks, and attitude judgments assessed with Likert-style responses.

Description of the Program. Up to three stimulus sets may be input for each batch of cards: a presentation set, the filler set, and the test set. A batch of cards is simply the card decks of one or more subjects. Ordinarily, if the presentation set is input, it matches the test set on some basis.

For each of the three stimulus sets, input to the program is divided into the part the subject will see and the part used to identify the stimulus during analysis. Currently, the stimulus itself must be 16 characters or less, and the identifying code is 4 characters. Both of these limitations can be changed with slight modifications to the program.

Each time the "RUN" command is encountered, the program will output a batch. The number of subjects per batch is controlled by the user. The user may optionally request that the test set be randomly permuted before each subject's deck is produced by the program. In this case, the presentation set is permuted into the same order as the test set. The program can produce any number of batches of decks. Any program specification may be changed from batch to batch.

The actual sequence of trials for the decks in a batch is specified according to a simple, free-field format. Individual stimuli are referred to by number, so that if the permutation option is used, the same numbers refer to different stimuli.

Practice runs of the program can be made that do not produce cards but do produce all other output of the program.

Output from the Program. All control cards are echoed, and an error-message facility is programmed into EXP. The program produces a key for each subject. If two or more subjects' decks are identical, only one key is produced.

After the decks have been put into appropriate piles corresponding to the subjects' responses, various fields on the cards are of use for sorting. There is a unique identifying number for each subject, and each card in each deck is labeled with the card (trial) number within that deck. There are also two fields on each card that can be used to identify the batch. Also, each stimulus from any set has a field associated with it that identifies it for analysis.

Any number of statistical packages or utilities can be used to sort the various piles by experiment, batch, subject, or trial type. For most applications, aggregation is performed so that the unit of analysis is the subject. But it is simple to analyze the data by item, or to look at conditional probabilities. In any case, SAS (Helwig \& Council, 1979) is especially useful for preparing the cards for statistical analyses. The SPSS AGGREGATE procedure (Nie, Hull, Jenkins, Steinbrenner, \& Bent, 1975) can also be used.

Computer and Language. The program is written in PL/I and has been compiled with the IBM PL/I optimizing $(X)$ compiler. EXP has been run extensively on an IBM 3033 computer running the MVS operating system with JES2.

Availability. The source to EXP and a user's guide are available from Charles Hofacker, Office of Academic 
Computing, University of California, Los Angeles, California 90024.

\section{REFERENCES}

Nie, N. H., Hull, C. H., Jenkins, J. G., Steinbrenner, K., \& BENT, D. H. Statistical package for the social sciences. New York: McGraw-Hill, 1975.

Helwia, J. T., \& Council, K. A. SAS user's guide. Raleigh, N.C: SAS Institute, 1979.

(Accepted for publication October 12, 1982.) 